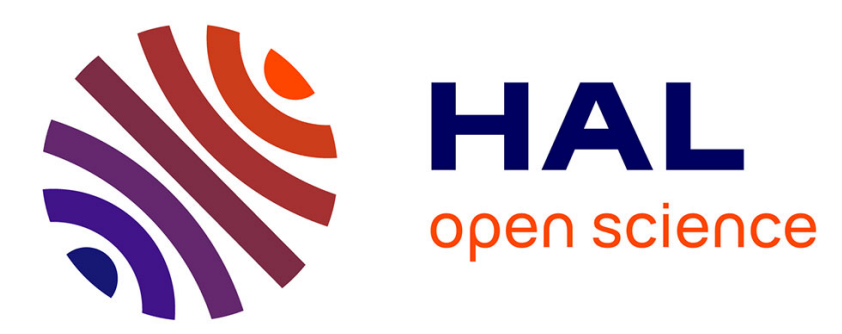

\title{
Implications of the O-GlcNAc modification in the regulation of nuclear apoptosis in $\mathrm{T}$ cells.
}

Bruno Johnson, Marlyse Opimba, Jacques Bernier

\section{To cite this version:}

Bruno Johnson, Marlyse Opimba, Jacques Bernier. Implications of the O-GlcNAc modification in the regulation of nuclear apoptosis in T cells.. BBA - Biochimica et Biophysica Acta, 2014, 1840 (1), pp.191-8. 10.1016/j.bbagen.2013.09.011 . hal-01177334

\section{HAL Id: hal-01177334 \\ https://hal.science/hal-01177334}

Submitted on 23 Jul 2015

HAL is a multi-disciplinary open access archive for the deposit and dissemination of scientific research documents, whether they are published or not. The documents may come from teaching and research institutions in France or abroad, or from public or private research centers.
L'archive ouverte pluridisciplinaire HAL, est destinée au dépôt et à la diffusion de documents scientifiques de niveau recherche, publiés ou non, émanant des établissements d'enseignement et de recherche français ou étrangers, des laboratoires publics ou privés. 


\section{Implications of the O-GIcNAc modification in the regulation of nuclear apoptosis in T cells}

- Bruno Johnson,

- Marlyse Opimba,

- Jacques Bernier.

\section{Abstract}

\section{Background}

$O$-linked $\beta-\mathrm{N}$-acetylglucosamine (O-GlcNAc) is a nutrient-/stress-sensitive posttranslational modification that affects nucleocytoplasmic proteins. The enzyme $O$ $\mathrm{N}$-acetylglucosamine transferase (OGT) catalyzes the addition of O-GlcNAc, whereas $\mathrm{O}-\mathrm{N}$-acetylglucosaminidase (OGA) removes it. $\mathrm{O}$-GlcNAcylation plays a role in fundamental regulatory mechanisms through the modification of proteins involved in cell division, metabolism, transcription, cell signaling and apoptosis. The effects of $O$-GlcNAcylation on apoptosis appear to be cell-dependent, as elevated levels played a protective role in primary neonatal rat ventricular myocytes but had a cytotoxic effect in rat pancreatic $\beta$-cells. The aim of the current study was to determine the implications of the O-GlcNAc modification on T cell apoptosis.

\section{Methods}

Human T lymphoblastic HPB-ALL cells were treated with the OGA inhibitor O-(2acetamido-2-deoxy-D-glucopyranosylidene) amino-N-phenylcarbamate (PUGNAc), or with glucosamine (GlcN), to increase O-GlcNAcylation. Apoptosis was induced in the presence of tributyltin (TBT). DNA fragmentation was observed by cell cycle analysis and corresponded to the sub G0/G1 population. O-GlcNAcylated proteins were detected by immunoblot using a specific antibody (ctd110.6) and were precipitated using succinylated wheat germ agglutinin (sWGA).

\section{Results}

HPB-ALL cells treated with PUGNAc displayed a significant reduction in DNA fragmentation after TBT-induced apoptosis. DFF45, the protein that inhibits the endonuclease DFF40, was identified to be O-GlcNAc modified. O-GlcNAcylated 
DFF45 appeared to be more resistant to caspase cleavage during apoptosis. Our results suggest that a decrease in the O-GlcNAc modification on DFF45 occurs before its cleavage by caspase.

\section{General significance}

Our results indicate that the $O$-GlcNAcylation of DFF45 may represent a mechanism to control the accidental activation of DFF.

Keywords

- Apoptosis;

- O-linked $\beta$-N-acetylglucosamine;

- O-(2-acetamidO-2-deoxy-D-glucopyranosylidene) amino-N-phenylcarbamate;

- TBT;

- DFF;

- DFF45

\section{Introduction}

Cancer cells require more energy than healthy cells to meet the requirements for their proliferation. Surprisingly, cancer cells generally display reduced rates of oxidative phosphorylation and generate most of their ATP through the less efficient process of fermentation, a phenomenon known as the "Warburg effect" [1]. Several studies have demonstrated that these cells require higher levels of intracellular glucose to produce sufficient levels of energy, as shown by the overexpression of GLUT transporters in most cancer cell lines [2], [3] and [4]. The impact of this altered glucose metabolism is not completely understood, but it has been speculated that cancer cells have evolved to produce energy through a pathway that increases their protection against apoptosis [5]. The higher influx of intracellular glucose in cancer cells can lead to the formation of uridine diphosphate n-acetylglucosamine (UDP-GlcNAc) through the hexosamine biosynthesis pathway.

The attachment of $\mathrm{N}$-acetylglucosamine ( $\mathrm{N}$-GlcNAc) monosaccharides is a nutrient-/stress-sensitive post-translational modification (PTM) that affects cytoplasmic and nuclear proteins [6] and [7]. The transfer of O-GlcNAc onto serine/threonine residues of target proteins ( $O$-GlcNAcylation) is catalyzed by the 
enzyme, $\mathrm{O}-\mathrm{N}$-acetylglucosamine transferase (OGT), whereas its removal is catalyzed by $\beta$-D-N-acetylglucosaminidase (OGA). The processes of $O$ GlcNAcylation and phosphorylation on serine/threonine residues compete and can co-regulate the activation of signaling pathways that had previously been described to be phospho-regulated [8]. Unlike phosphorylation, the impact of $O$ GlcNAcylation on apoptosis is unclear and has not been thoroughly studied. An increase in this type of $O$-glycosylation in glucose-stimulated rat pancreatic $\beta$ cells correlated with an increase in apoptosis [9]. As shown in murine pancreatic $\beta$-cells, this effect could be due to the O-GlcNAcylation of Akt1, thereby preventing its phosphorylation and activation by mTORC2 and leading to apoptosis [7]. In contrast, an increase in O-GlcNAc was found to promote cell survival in other studies; in primary cultures of neonatal rat ventricular myocytes, glucosamine increased $O$-GlcNAc levels and protected the cells from ischemiareperfusion injuries. The glucosamine treatment increased mitochondrial levels of anti-apoptotic $\mathrm{Bcl}-2$, altered p38 activation and reduced calpain proteolysis [10], [11], [12] and [13]. Furthermore, O-GlcNAcylation protected several cell lines from numerous types of stress stimuli, including heat, toxic agents, UVB rays, glucose deprivation and ischemia-reperfusion injuries [14], [15] and [16]. Taken together, the effects of O-GlcNAcylation on apoptosis appear to be cell type-dependent, and not every cell type has been studied thus far. There is also an apparent lack of knowledge of the impact of this type of $O$-glycosylation on nuclear apoptosis.

DNA fragmentation factor 40 (DFF40/CAD/CPAN) is the principal endonuclease responsible for oligosomal fragmentation of DNA during nuclear apoptosis. In healthy cells, DFF40 is bound to its inhibitor, DFF45/ICAD, in an inactive heteromeric complex[17] and [18]. During apoptosis, effector caspases 3 and 7 cleave DFF45 to release DFF40, which oligomerizes and cleaves the DNA [18]. In this report, we evaluated the effects of O-GlcNAcylation on the induction of apoptosis in T cells. O-(2-acetamidO-2-deoxy-D-glucopyranosylidene) amino-Nphenylcarbamate (PUGNAc), a potent inhibitor of OGA, was used to increase $O$ GlcNAcylation. Our results demonstrated that increasing the O-GlcNAc modification in HPB-ALL cells had no protective effect against the induction of apoptosis by the mitochondrial disrupting agent, tributyltin (TBT). However, a significant decrease in DNA fragmentation occurred in PUGNAc-treated cells. The O-GlcNAcylation of DFF45 may represent a mechanism to inhibit the accidental activation of DFF40, which can be deleterious to the organism. 


\section{Materials and methods}

\subsection{Materials}

RPMI 1640 (R8758), fetal bovine serum (F1051), penicillin/streptomycin (P0781), HEPES (H0887), D-(+)-glucosamine (GlcN, G4875), a monoclonal anti-O-linked $\beta$-N-acetylglucosamine antibody (ctd110.6, O7764) and TBT chloride $(96 \%$ purity, T50202) were purchased from Sigma-Aldrich Corporation (Milwaukee, WI, USA). Anti-caspase-3 (9662) was from Cell Signaling Technology Inc. (Danvers, MA, USA). The anti-DFF45 antibody used for Western blotting was from Prosci Inc. (Poway, CA, USA). The anti-DFF45 antibody used for immunoprecipitation (AB16961) and Pure Proteome magnetic beads (LSKMAGT10) were purchased from EMD Millipore Corporation (Billerica, MA, USA). Anti-mouse IgM (sc-2064) was purchased from Santa Cruz Biotechnology, Inc. (Santa Cruz, CA, USA) and anti-rabbit IgG was obtained from New England Biolabs Ltd. (Whitby, ON, Canada). sWGA-bound agarose beads were from Vector Laboratories Inc. (Burlington, ON, Canada). Z-VAD-FMK (ab120382) were from Abcam Inc. (Toronto, ON, Canada). SuperSignal West Femto chemiluminescent substrate (34096) were purchased from Thermo Scientific Pierce (Rockford, IL, USA). Destreak buffer, IPG buffer and the Ettan IPGPhor system were all obtained from GE Healthcare Sciences (Mississauga, ON, Canada). A FACScan ${ }^{\circledR}$ from Becton Dickinson (Oakville, ON, Canada) was used. EtBr was visualized by UV transillumination using a Fluor S-Multilmager from Bio-Rad Laboratories, Ltd. (Mississauga, ON, Canada).

\subsection{Cell culture}

The HPB-ALL human T lymphoma cell line was provided by the American Type Culture Collection (Manassas, VA, USA). The cells were grown in RPMI 1640 supplemented with $10 \%$ heat-inactivated fetal bovine serum, $100 \mathrm{U} / \mathrm{ml}$ penicillin, $100 \mu \mathrm{g} / \mathrm{ml}$ streptomycin (Sigma-Aldrich Corporation, P0781) and $10 \mathrm{mM} \mathrm{HEPES}$ when required.

\subsection{Cell treatment}

Cells were treated as previously described with some modifications [19] and [20]. Briefly, the cells were washed thrice in phosphate-buffered saline (PBS) and resuspended at a density of $1 \times 10^{6} \mathrm{cells} / \mathrm{ml}$ in RPMI 1640 supplemented with $0.1 \%$ (4-h incubation) or $1 \%$ (16-h incubation) heat-inactivated fetal bovine 
serum. The cells were treated or not with $75 \mu \mathrm{M}$ O-(2-Acetamido-2-deoxy-Dglucopyranosyl iden amino) $N$ phenyl carbamate (PUGNAc), $5 \mathrm{mM} \mathrm{GlcN}$ or $16 \mathrm{mM}$ for $16 \mathrm{~h}$ at $37^{\circ} \mathrm{C}$. The cells were exposed to $0.8 \mu \mathrm{M}$ TBT (tributyltin chloride, 96\% purity, Sigma-Aldrich Corporation, Milwaukee, WI, USA) diluted in ethanol and incubated for $4 \mathrm{~h}$ at $37^{\circ} \mathrm{C}$. Control cells were exposed to the TBT vehicle, $\mathrm{EtOH}(0.1 \%)$.

\subsection{Apoptosis assessment}

Internucleosomal DNA fragmentation was evaluated by cell cycle analysis using the G1/G0 subdiploid peak quantification as described previously with some modifications[20]. Briefly, the treated cells were washed once and resuspended at a density of $1 \times 10^{6} \mathrm{cells} / \mathrm{ml}$ in PBS. Nine volumes of $70 \% \mathrm{EtOH}$ were then slowly added for fixation. The cells were incubated at $-20^{\circ} \mathrm{C}$ overnight and then washed once in PBS and resuspended at $3.33 \times 10^{6} \mathrm{cells} / \mathrm{ml}$ in modified Krishan buffer $(0.1 \%$ sodium citrate, $0.3 \% \mathrm{NP}-40,20 \mu \mathrm{g} / \mathrm{ml}$ RNase and $50 \mu \mathrm{g} / \mathrm{ml}$ propidium iodide). The samples were incubated for $1 \mathrm{~h}$ at $37^{\circ} \mathrm{C}$ and analyzed by flow cytometry on a FACScan ${ }^{\circledR}$. Analyses were performed using live gates to discriminate between doublets and cells exhibiting reduced DNA content corresponding to the sub $\mathrm{G} 1 / \mathrm{G} 0$ diploid peak; the latter cells were defined as apoptotic cells. The results were confirmed by evaluation of internucleosomal DNA fragmentation performed by standard agarose gel electrophoresis as described previously [21]. Briefly, $1.5 \times 10^{6}$ cells were resuspended in $1 \mathrm{ml}$ PBS and added drop by drop to $9 \mathrm{ml}$ of cold $70 \%$ ethanol for fixation. Samples were stored at $-20^{\circ} \mathrm{C}$ for $24-72 \mathrm{~h}$, centrifuged at $800 \mathrm{~g}$ for $5 \mathrm{~min}$, resuspended in $40 \mathrm{ul}$ of phosphate-citrate buffer, consisting of $0.2 \mathrm{M} \mathrm{Na}_{2} \mathrm{HPO}_{4}$ and $0.1 \mathrm{M}$ citric acid pH 7.8 (192:8) and incubated at room temperature for $30 \mathrm{~min}$. Afterward, $0.02 \%$ Nonidet NP-40 and $0.08 \mathrm{mg} / \mathrm{ml}$ Rnase A was added. Cells were incubated $30 \mathrm{~min}$ at $37^{\circ} \mathrm{C}$ and $0.08 \mathrm{mg} / \mathrm{ml}$ proteinase $\mathrm{K}$ was added before an additional 30 min of incubation at $37^{\circ} \mathrm{C}$. Extracts were then diluted in $33.3 \%(\mathrm{v} / \mathrm{v})$ loading buffer (10 mM EDTA, pH 8.0, 1.0\% (w/v) low-gelling $\mathrm{T}^{0}$ agarose, $0.25 \%(\mathrm{w} / \mathrm{v})$ bromophenol blue and $40 \%$ (w/v) sucrose). Samples were loaded onto a $2 \%$ $(\mathrm{w} / \mathrm{v})$ agarose gel. Migration was conducted overnight at $10 \mathrm{~V}$ in TBE $0.5 \times$ buffer. The DNA ladder was stained with $0.5 \mu \mathrm{M} \mathrm{EtBr}$ and visualized by UV transillumination using a Fluor S-Multilmager (Bio-Rad Laboratories, Ltd., Mississauga, ON, Canada). 


\subsection{Immunoprecipitation and Western blot analysis}

The immunoprecipitation of O-GlcNAcylated proteins was performed with $20 \times 10^{6}$ treated cells for each condition. After harvesting, the cells were washed once in PBS, resuspended in lysis buffer (10 mM Tris-HCl, pH 7.5, 1 mM EGTA, $1 \%$ Triton X-100 and $1 \times$ of the Halt protease inhibitor cocktail) and incubated for $30 \mathrm{~min}$ at $4{ }^{\circ} \mathrm{C}$ with vortexing every $10 \mathrm{~min}$. Cell lysates were centrifuged at $13,000 \times g$ for $5 \mathrm{~min}$ at $4{ }^{\circ} \mathrm{C}$. The supernatants were mixed by rotation for $1 \mathrm{~h}$ at $4{ }^{\circ} \mathrm{C}$ with agarose beads coupled to sWGA. The samples were centrifuged at $13,000 \times g$ for $1 \mathrm{~min}$ at $4{ }^{\circ} \mathrm{C}$ and washed five times with lysis buffer. The $O$ GlcNAcylated proteins coupled to beads were dissociated by boiling the samples at $100{ }^{\circ} \mathrm{C}$ for $10 \mathrm{~min}$ in $2 \times$ Laemmli buffer. The proteins were loaded onto a $10 \%$ SDS-polyacrylamide gel, separated and transferred onto a PVDF membrane. Membranes were blocked with $0.3 \% \mathrm{BSA}$ for $1 \mathrm{~h}$ at room temperature and probed overnight at $4{ }^{\circ} \mathrm{C}$ with the appropriate primary antibody and $1 \mathrm{~h}$ at room temperature with the corresponding secondary antibody. Bands were visualized by chemiluminescence. The immunoprecipitation of DFF45 was performed with a direct IP kit. $40 \times 10^{6}$ cells were washed and lysed as described above. As according to the manufacturer's instructions, $50 \mu$ of magnetic beads coupled to $G$ protein were washed thrice and resuspended in PBS-T. $4 \mu \mathrm{g}$ of $\alpha$-DFF45 was added to the beads and allowed to fix for $10 \mathrm{~min}$ at room temperature. Cells lysates were centrifuged at $13,000 \times g$ for $10 \mathrm{~min}$. The supernatants were transferred to the $\alpha$-DFF45-coupled beads and mixed by rotation overnight at $4{ }^{\circ} \mathrm{C}$. Beads were washed thrice with PBS-T, Laemmli buffer was added, samples were boiled for $10 \mathrm{~min}$ and saved for Western blotting. Densitometric analyses were performed using ImageJ $1.44 p$ software (NIH, MD, USA).

\section{6. $2 \mathrm{D}$ gel electrophoresis}

HPB-ALL cells $\left(20 \times 10^{6}\right)$ were exposed to GlcN $(16 \mathrm{mM})$ for $16 \mathrm{~h}$. The cells were harvested in IP lysis buffer (50 mM Tris- $\mathrm{HCl}, \mathrm{pH} 8.0,150 \mathrm{mM} \mathrm{NaCl}, 0.02 \%$ sodium azide, $1 \%$ NP-40 and $1 \times$ of the Halt protease inhibitor cocktail), and total $O$-GlcNacylated proteins were immunoprecipitated using agarose beads coupled to sWGA. The beads were washed and resuspended in Destreak rehydration buffer supplemented with $60 \mathrm{mM}$ DTT and 0.5\% IPG buffer (pH 310). The samples were mixed by rotation at room temperature for $30 \mathrm{~min}$, and the supernatant obtained after centrifugation was loaded onto a 13-cm IPG strip 
$(\mathrm{pH} 3-10)$. The samples were rehydrated for $15 \mathrm{~h}$ at $30 \mathrm{~V}$, and isoelectric focusing was performed on an Ettan IPGPhor system according to the manufacturer's instructions. Second dimension was performed on a $10 \% \mathrm{gel}$, and the proteins were transferred onto a nitrocellulose membrane. The proteins were identified by Western blot as previously described. Destreak buffer, IPG buffer and the Ettan IPGPhor system were all obtained from GE Healthcare Sciences (Mississauga, ON, Canada).

\subsection{Caspase-3 cleavage assay}

HPB-ALL cells $\left(5 \times 10^{6}\right)$ were treated or not with GlcN $(16 \mathrm{mM})$ for $16 \mathrm{~h}$, and the cells were washed once in PBS and resuspended in lysis buffer. Caspase-3 treatment was performed as previously described with some modifications [22]. Briefly, $25 \mu \mathrm{g}$ of protein in lysis buffer was diluted 1:10 (V/V) in caspase buffer (50 mM HEPES pH 7.4, $100 \mathrm{mM} \mathrm{NaCl}, 1 \mathrm{mM}$ EDTA, 10\% glycerol and 0.1\% NP40). Two units of caspase-3 (ab52101, Abcam Inc., Toronto, ON, Canada) and/or $40 \mu \mathrm{M}$ of Z-VAD-FMK (ab120382, Abcam Inc., Toronto, ON, Canada) were added, and the samples were rotated for $2 \mathrm{~h}$ at $37^{\circ} \mathrm{C}$. Laemmli buffer $(4 \times)$ was added, and the samples were boiled for $10 \mathrm{~min}$. DFF45 cleavage was analyzed as described before.

\subsection{Statistical analysis}

Experimental data were expressed as the means \pm SEM. A repeated-measures ANOVA (Dunnett's test) was performed using GraphPad Prism software (version 5.01). Differences were considered statistically significant when $p<0.05$.

\section{Results}

\subsection{O-N-acetylglucosaminylation in HPB-ALL cells reduced TBT-induced DNA fragmentation}

To determine the effects of $O$-GlcNAcylation on TBT-induced apoptosis, human HPB-ALL T cells were treated or not with PUGNAc for $18 \mathrm{~h}$. PUGNAc is an inhibitor of $O$-GlcNAcase and was used to increase $O$-GlcNAcylation. Thereafter, the cells were exposed to TBT for $4 \mathrm{~h}$ and cleavage of pro-caspase-3 was determined. As shown inFig. 1A, the presence of PUGNAc and GlcN alone did not cause pro-caspase 3 cleavage, indicating that the concentrations used were not cytotoxic. This result was confirmed by Trypan blue exclusion staining (data not shown). In the presence of TBT, pro-caspase-3 bands disappeared and 
caspase 3 was activated. PUGNAc- plus TBT-treated cells were also undergoing apoptosis, as the processing of pro-caspase-3 was observed. Next, we analyzed the late event of apoptosis, DNA fragmentation, by performing a sub G0/G1 cell cycle analysis using flow cytometry ( Fig. 1B). The results showed that there was more DNA fragmentation in the cells exposed to TBT (50.13\%) compared with the control cells $(11.7 \%)$. In contrast, the inhibition of $O$-GlcNAcase with PUGNAc significantly reduced the number of cells in sub G0/G1 (37.0\%), suggesting that an increase in O-GlcNAc decreases DNA fragmentation. To confirm this observation, DNA laddering was examined on $2 \%$ agarose gels. The presence of PUGNAc and GIcN in TBT-treated cells reduced the intensity of the DNA bands, thereby confirming a reduction in internucleosomal DNA fragmentation ( Fig. 1C). 


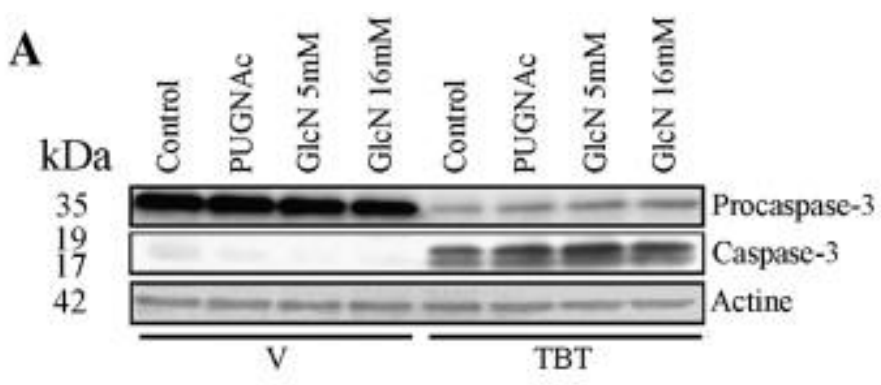

B
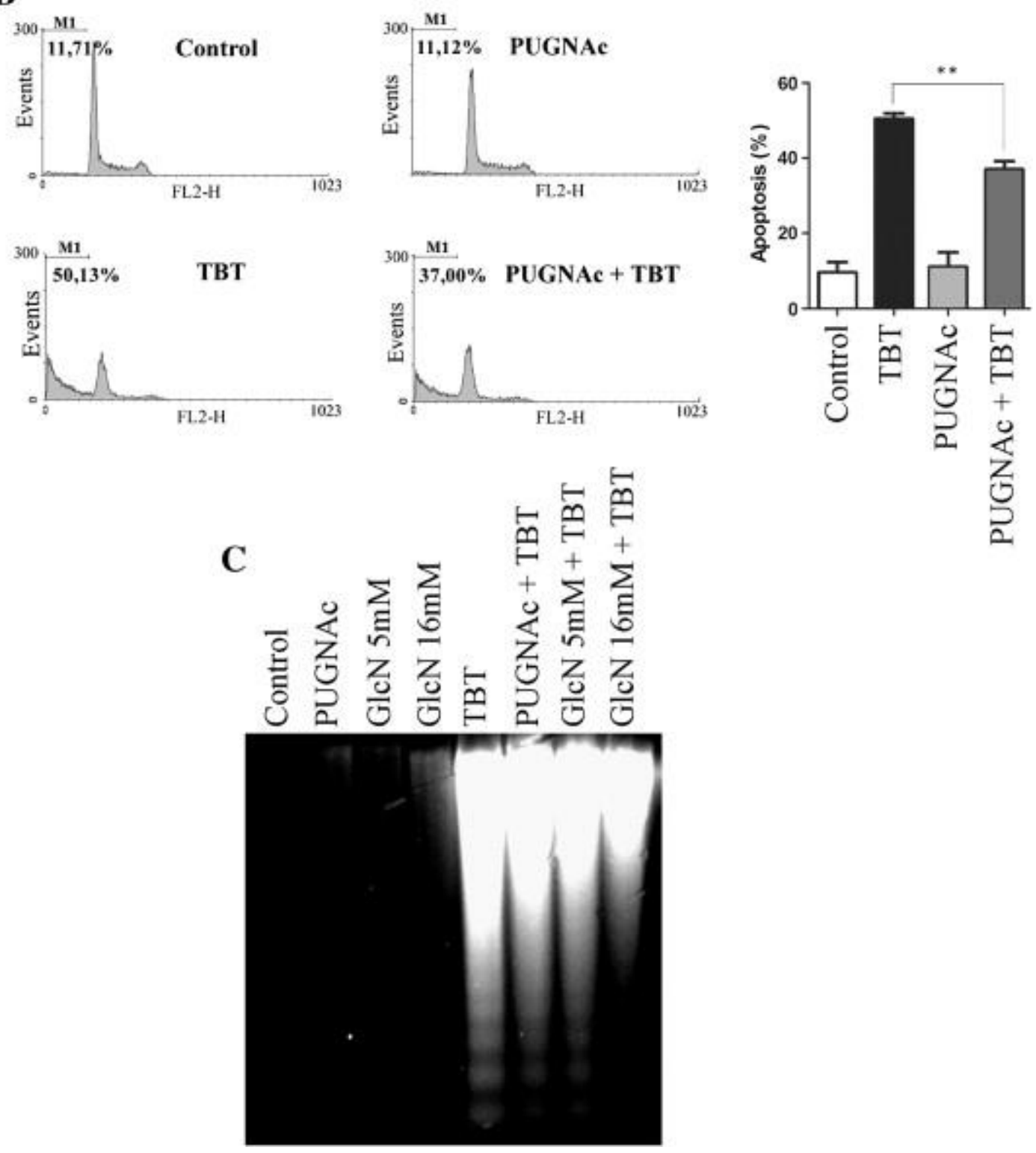

Fig. 1.

Effects of $O$-glycosylation levels on the induction of apoptosis. HPB-ALL cells were exposed or not to PUGNAc $(75 \mu \mathrm{M})$ for $16 \mathrm{~h}$ and treated or not with TBT $(0.8 \mu \mathrm{M})$ for $4 \mathrm{~h}$ at $37^{\circ} \mathrm{C}$. Control cells were incubated with $0.1 \%(\mathrm{v} / \mathrm{v}) \mathrm{EtOH}$. A) Proteins were extracted, 
loaded onto 10\% SDS-PAGE gels and immunoblotted with an antibody that detects full length caspase-3 (35 kDa) and its activated forms (17 and $19 \mathrm{kDa})$. B) After labeling with $\mathrm{PI}$, cells were analyzed by flow cytometry, and apoptotic cells were identified as those exhibiting reduced DNA content (M1). One way Anova with Dunnett's post-hoc test was performed $\left({ }^{* *} \mathrm{P}<0.01\right)$. C) DNA was extracted, and internucleosomal fragmentation was visualized on a $2 \%$ agarose gel. All data are representative of at least 3 independent experiments.

Figure options

3.2. Apoptosis was associated with the modulation of $O$-GlcNAcylated proteins To determine the $O$-GlcNAcylation of proteins during TBT-induced apoptosis in HPB-ALL cells, O-GlcNAc modifications were analyzed in total cell lysates by Western blot (Fig. 2). To confirm the specificity of detection using the ctd110.6 antibody, the cells were treated with glucosamine (GlcN) before exposure to TBT. Also, the CTD110.6 was preadsorbed with GlcNAc on one membrane, revealing the existence of only 2 non-specific bands. GlcN was used to induce elevated $O$-GlcNAcylation through the hexosamine biosynthesis pathway. Densitometry analysis confirmed a global increase inO-GlcNAcylation in apoptotic cells as compared with control cells, although some bands disappeared in the TBT-treated cells. GlcN treatment increased the detection of O-GlcNAc modifications, and similar patterns were observed in treated or untreated cells, thereby validating the method of detection of $O$-GlcNAcylated proteins. Interestingly, in TBT-induced apoptosis, a band at $45 \mathrm{kDa}$ appeared to be partially deglycosylated, whereas a band at approximately $200 \mathrm{kDa}$ seemed to be totally deglycosylated. The decrease in O-GlcNAcylation of the 45-kDa band in the TBT-treated cells in the presence of GlcN was reduced, suggesting that the nutritional environment influences the $O$-GlcNAcylation of cells. The O-GlcNAc modification is found on many nuclear and cytoplasmic proteins and is a highly dynamic post-translational modification that plays a key role in signal transduction pathways. Next, we determined the level of $O$-GlcNAc modification

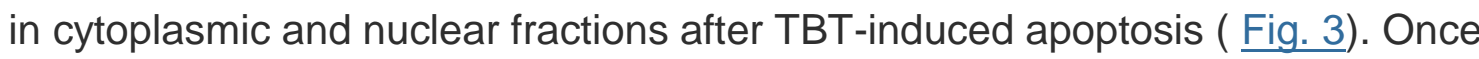
again, samples were compared to PUGNAc-treated cells to validate the specificity of the CTD110.6 antibody. Modulations in the expression or the cellular localization of a 45-kDa band was observed again and this, in both fractions ( Fig. 3C). As seen on total cell lysates, our results clearly show an increase in total $O$-GlcNAcylated proteins in the nucleus and cytoplasm of apoptotic cells. 

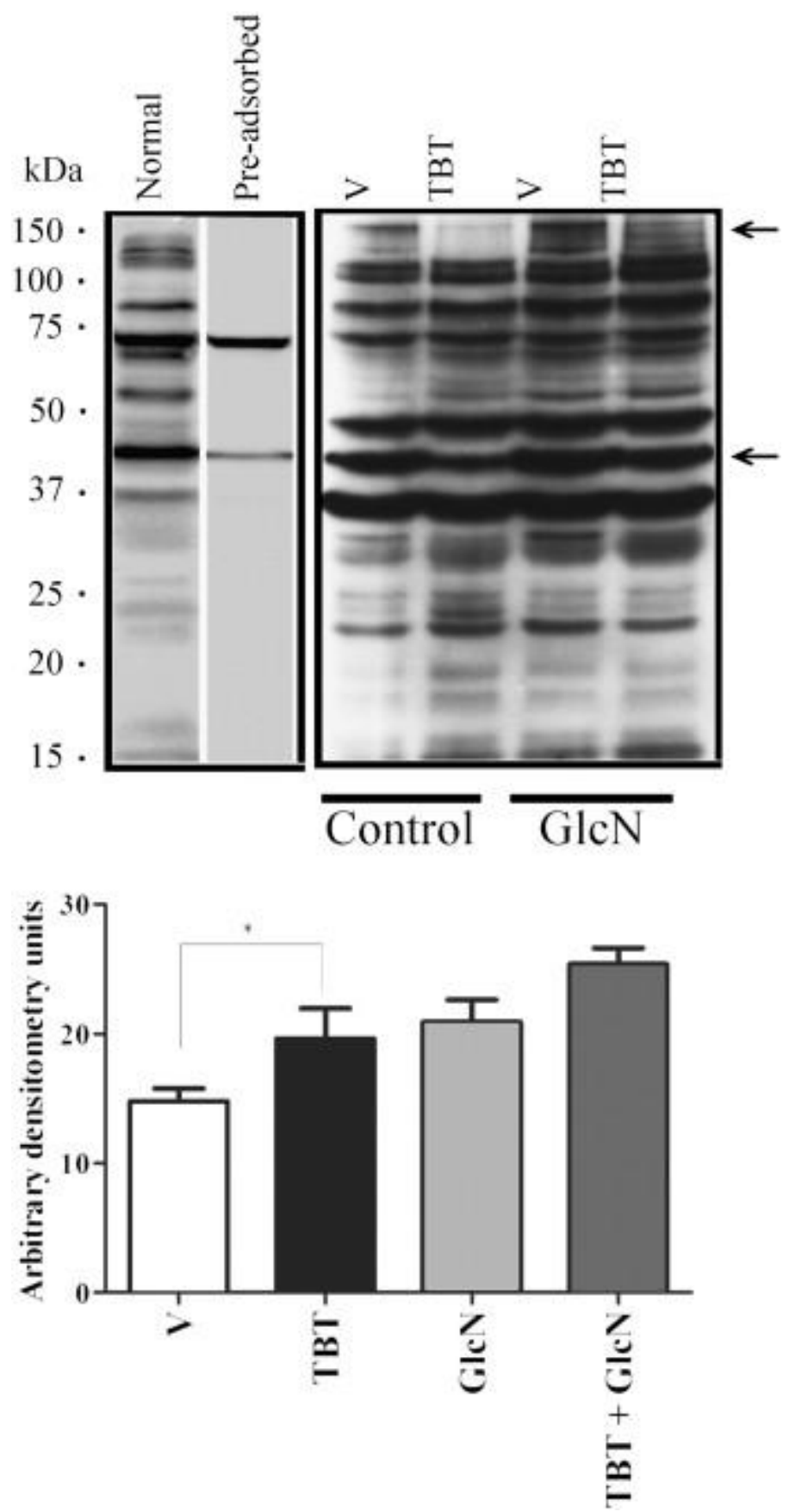

Fig. 2.

Modulation of $O$-GlcNAC levels in apoptotic cells. Cells were exposed or not to glucosamine (GlcN, $16 \mathrm{mM})$ for $16 \mathrm{~h}$ and treated with TBT $(0.8 \mu \mathrm{M})$ for $4 \mathrm{~h}$ at $37^{\circ} \mathrm{C}$.

Proteins were extracted, loaded onto $10 \%$ SDS-PAGE gels and immunoblotted with an $\alpha$ O-GlcNAcylated protein antibody (ctd110.6). To determine the specificity of detection of O-GlcNAcylated proteins, two samples of GlcN-treated cells were compared and the CTD110.6 antibody was preadsorbed with $O-\mathrm{N}$-acetylglucosamine $(20 \mathrm{mmol} / \mathrm{L})$ for $1 \mathrm{~h}$ on one of the membranes. Control cells were incubated with $0.1 \%(\mathrm{v} / \mathrm{v}) \mathrm{EtOH}$. All data are 
representative of at least 3 independent experiments. Densitometry of total lanes were adjusted to actin expression and one way Anova with Dunnett's post-hoc test was performed ( ${ }^{*} P<0.05$ ).

Figure options
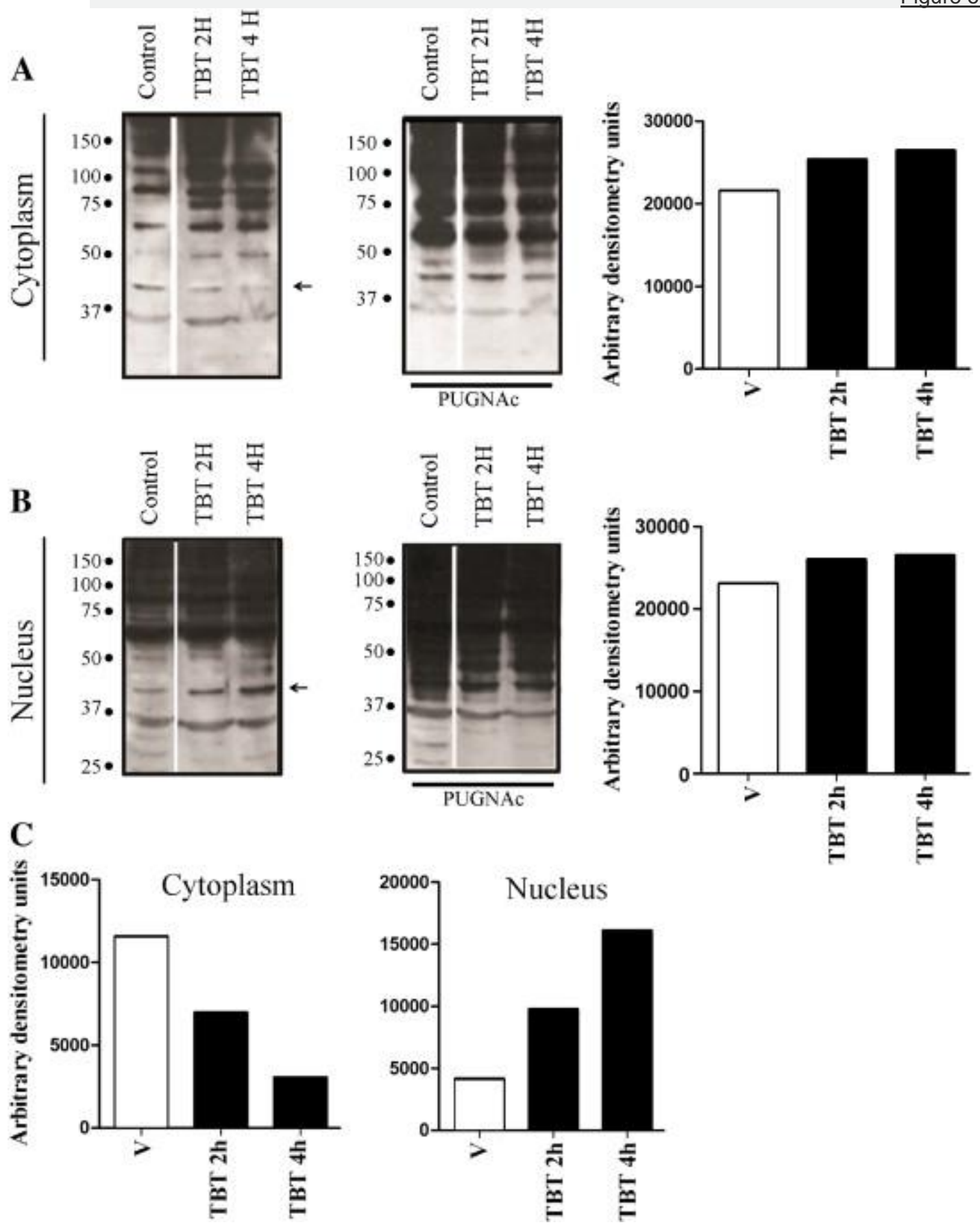

Fig. 3.

Change in O-GIcNAC modifications in both cytoplasmic and nuclear fractions of apoptotic cells. Cells were exposed to PUGNAc $(75 \mu \mathrm{M})$ for $16 \mathrm{~h}$ and treated with TBT $(0.8 \mu \mathrm{M})$ for 
0,2 and $4 \mathrm{~h}$ at $37^{\circ} \mathrm{C}$. Cytoplasmic (A) and nuclear (B) proteins were extracted and loaded onto $10 \%$ SDS-PAGE gels and immunoblotted with an $\alpha-O-G l c N A c y l a t e d$ protein antibody (ctd110.6). Densitometry of total lanes of the shown Western blot images are presented to facilitate the interpretation. C) Densitometry analysis of the identified $45 \mathrm{kDa}$ band in A and B. Control cells were incubated with $0.1 \%(\mathrm{v} / \mathrm{v}) \mathrm{EtOH}$. All data are representative of at least 3 independent experiments.

Figure options

\subsection{DFF45 was subject to the O-GIcNAc modification}

The next step was to determine whether the 45-kDa band corresponded to DFF45, the inhibitory protein of the DFF complex. HPB-ALL cells were treated or not with GlcN plus PUGNAc, and total O-GlcNAc-modified proteins were precipitated with succinylated wheat germ agglutinin (sWGA), a lectin binding specifically to $O$-GlcNAc, coupled to agarose beads. The proteins were then loaded and separated on a $10 \%$ polyacrylamide gel, transferred on a PVDF membrane and probed with an anti-DFF45 antibody. As shown in Fig. 4A, DFF45 was precipitated with sWGA and thus was $O$-GlcNAcylated in healthy cells. Treatment of cells with GIcN and PUGNAc increased the level of DFF45 in the sWGA-precipitated fraction, demonstrating its $O$-GlcNAcylation. Next, we immunoprecipitated DFF45 and determined the presence of the O-GIcNAc modification. As expected, a 45-kDa band, corresponding to DFF45, was $O$ GlcNAcylated. To confirm the identity of DFF45, we performed a 2D gel analysis. HPB-ALL cells were treated with GlcN overnight, and O-GlcNAcylated proteins were precipitated using sWGA. The $O$-GIcNAc-modified proteins were released from the lectin and separated in 1D by isoelectric focusing followed by a second separation on a $10 \%$ SDS-PAGE gel. DFF45 spots were detected by Western blot. Two spots were detected at approximately 45 and $75 \mathrm{kDa}$ ( Fig. 4B). The spot at $45 \mathrm{kDa}$ had an isoelectric point with a theoretical value of 4.68 for DFF45 [23]. Thus, DFF45 is subject to the O-GlcNAc modification. 
A

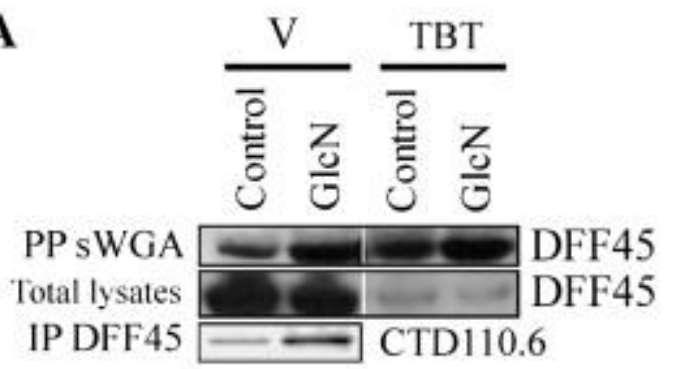

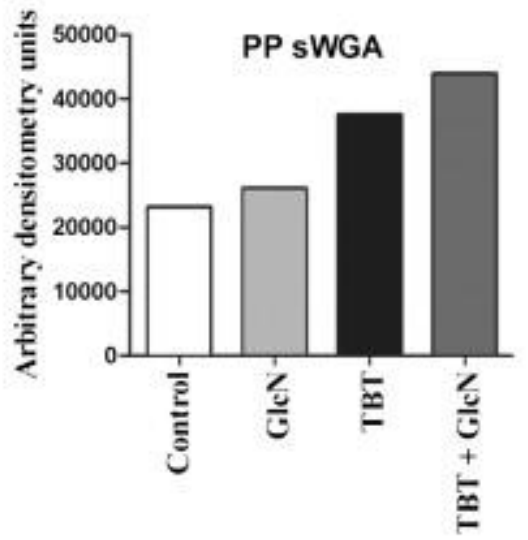

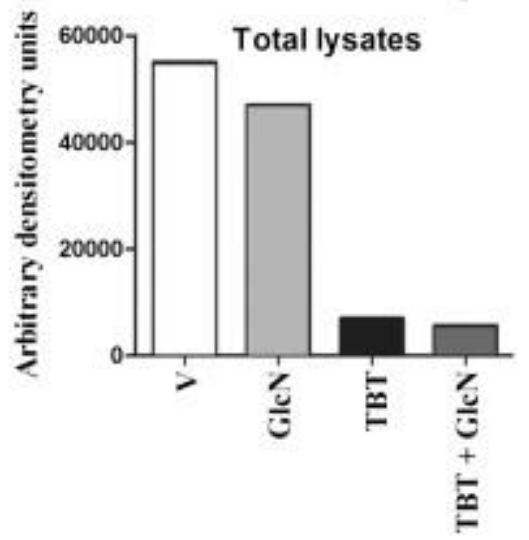

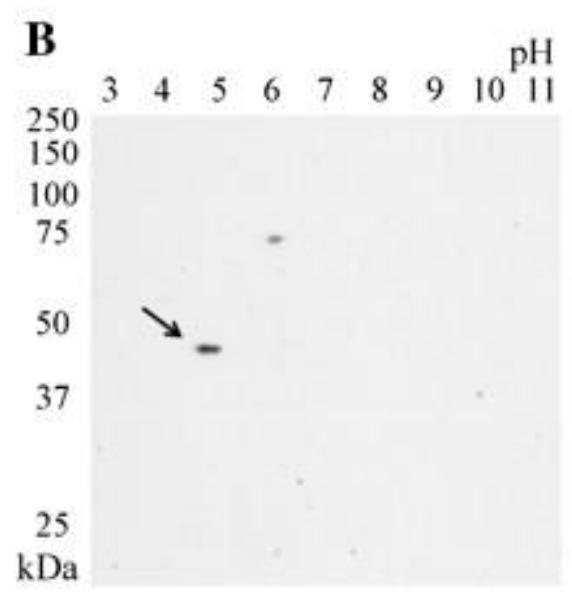

C

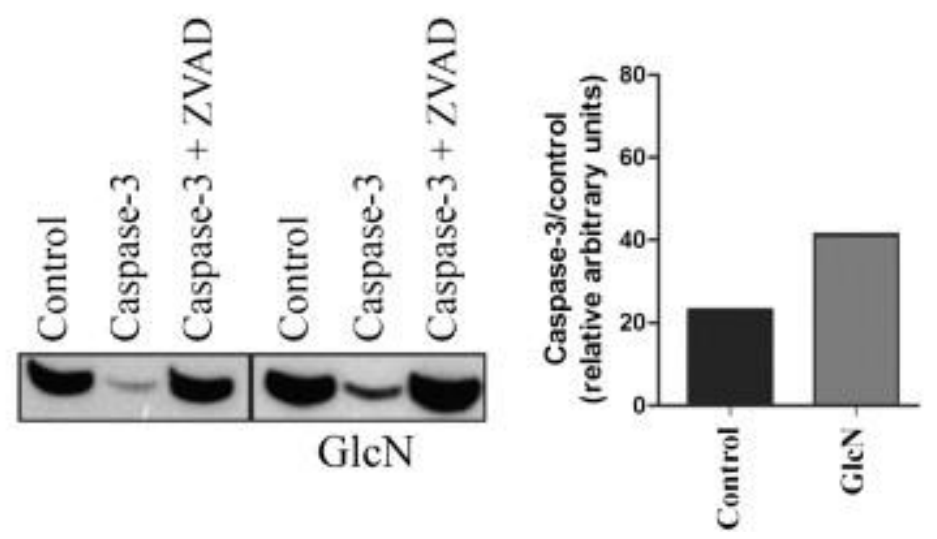

Fig. 4.

Identification and modulation of DFF45 O-glycosylation in HPB-ALL cells. A) Cells were exposed or not to GlcN (16 mM) and PUGNAc $(10 \mu \mathrm{M})$ for $16 \mathrm{~h}$ and treated or not with TBT $(0.8 \mu \mathrm{M})$ for $4 \mathrm{~h}$ at $37^{\circ} \mathrm{C}$. Cells were harvested, and total $O-G l c N a c y l a t e d$ proteins were precipitated using agarose beads coupled to sWGA, or DFF45 was immunoprecipitated with a specific antibody. Samples were loaded onto a $10 \%$ polyacrylamide gel and immunoblotted with an $\alpha$-DFF45 or 110.6 antibody. B) HPB-ALL cells were exposed to GlcN (16 mM) for $16 \mathrm{~h}$, and total O-GlcNacylated proteins were immunoprecipitated using sWGA. Proteins were resuspended in Destreak rehydration buffer and loaded onto a 13-cm IPG strip ( $\mathrm{pH} 3-10)$. Isoelectric focusing was performed using the Ettan IPGPhor system. Second dimension was performed on a 10\% SDS-

PAGE gel. Proteins were transferred onto a nitrocellulose membrane and immunoblotted with an $\alpha$-DFF45 antibody. C) $5 \times 10^{6}$ cells exposed or not to GlcN (16 mM) were harvested, lysed and were exposed or not to caspase-3 and ZVAD for $2 \mathrm{~h}$. Samples were loaded onto a $10 \%$ polyacrylamide gel and immunoblotted with an $\alpha$-DFF45 antibody. All 
data are representative of at least 3 independent experiments. Densitometry of shown immunoblots are presented.

Figure options

3.4. O-GlcNAc modifications protect DFF45 from caspase cleavage during apoptosis

A small portion of DFF45 is O-GlcNAcylated in normal cells. Treatment with GlcN and PUGNAc increased this modification. Moreover, treatment of HPB-ALL cells with PUGNAc decreased DNA fragmentation after exposure to a mitochondrial perturbing agent. We next determined whether this type of modification could protect DFF45 from caspase cleavage upon TBT-induced apoptosis. Cells were treated or not with GlcN plus PUGNAc, and apoptosis was induced with TBT. The cells were lysed after $4 \mathrm{~h}, \mathrm{O}$-GlcNAc-modified proteins were precipitated with sWGA and the presence of DFF45 was determined by Western blot.

Densitometry in Fig. 4A showed that the level of DFF45 protein in control or GIcN- plus PUGNAc-treated cells remained similar between non-apoptotic (vehicle-treated) and apoptotic (TBT-treated) cells, suggesting that O-GlcNAcmodified DFF45 was not cleaved following TBT-induced apoptosis. Total cell lysates $(50 \mu \mathrm{g})$ analyzed for the presence of DFF45 showed that the majority of DFF45 disappeared after $4 \mathrm{~h}$ of TBT treatment. The remaining uncleaved DFF45 may represent the $O$-GlcNAc-modified fraction that was enriched by sWGA precipitation.

Caspase-3 is involved in the processing of DFF45 during apoptosis. We hypothesized that $O$-GlcNAcylated DFF45 was protected from caspase-3 cleavage. To test this hypothesis, cells were treated or not with GlcN, and apoptosis was induced with TBT for $4 \mathrm{~h}$. The cells were lysed and a aliquot of total protein treated with active caspase-3 with/without the caspase inhibitor, ZVAD-FMK, for $2 \mathrm{~h}$ and the presence of DFF45 was determined by Western blot. The increase in the O-GlcNAcylation of DFF45 by GlcN treatment resulted in a partial resistance to caspase-3 cleavage ( Fig. 4C). Densitometric analysis shown an increase of two fold of DFF45 in GlcN treated cells as compared to untreated cells. Thus, the O-GlcNAcylated form of DFF45 is more resistant to caspase-3 cleavage, although this modified form of DFF45 seems to be poorly represented in apoptotic cells.

\section{Discussion}


The post-translational addition of $O$-GlcNAc is primarily implicated in the modulation of cell signaling and transcriptional regulatory pathways in response to nutrients and stress. The attachment of the monosaccharide to Ser and/or Thr residues occurs mainly on nuclear and cytosolic proteins [24]. An increase in $O$ GlcNAc levels has been shown to be protective against stress or trauma in vivo [25] and [26]. In the current study, we found that an increase in $O$ GlcNAcylation occurred in apoptotic T cells. Using PUGNAc, a potent inhibitor of OGA, we showed that an increase in O-GlcNAcylation protected cells from TBTinduced DNA fragmentation without any effects on caspase-3 activation (Fig. 2). Previous studies have reported that $O$-GlcNAc also protects against apoptosis induced by ischemia-reperfusion injury in neonatal cardiomyocytes [10]. In the latter cells, an increase in $O$-GlcNAcylation was found to reduce calpain proteolysis, alter p38 activation and increase mitochondrial Bcl-2 levels. In contrast, other studies have found that an increase in O-GlcNAcylation increases apoptosis. In murine $\beta$-pancreatic cells, the hyperglycemic condition increased the O-GlcNAc modification of Ser473 and decreased the phosphorylation of Akt1, thereby inhibiting the anti-apoptotic pathway controlled by this protein [7]. However, Ku and colleagues found that the O-glycosylation of keratin 18 (K18) induced Akt1 activation and favored cell survival, indicating that this pathway is heavily regulated [27]. Although PUGNAc did not provide any protection from apoptosis, a significant decrease in DNA fragmentation was observed in our study (Fig. 1).

O-GlcNAc modifications of serines/threonines on cytoplasmic and nuclear proteins are involved in cell cycle control, cell development and cell apoptosis. We showed that $O$-GlcNAcylation was predominantly increased during apoptosis ( Fig. 2). Some decrease in the modification was also observed, particularly of a band approximately $45 \mathrm{kDa}$ in size. An analysis of cytoplasmic and nuclear fractions showed that the $45-\mathrm{kDa}$ band decreased in the cytoplasm and increased in the nucleus ( $\underline{\text { Fig. } 3}$ ). This observation suggests two possibilities; $O$ GlcNAcylation is involved in either the nucleocytoplasmic translocation of proteins or the degradation of proteins. O-GlcNAcylation has been shown to affect ELF-1 translocation, a process that regulates the transcription of several hematopoietic genes [28]. Using an OGA inhibitor and glucosamine to increase total O-GlcNAcylation levels, we identified DFF45, the inhibitory protein of DFF40 in the DFF complex, as an O-GlcNAc-modified protein. In a study on global identification of $O$-GlcNAc-modified proteins, the DFF45 was identified as an $O$ - 
GlcNAc-modified protein in non-apoptotic HeLa cells [29]. Interestingly, cleavage of DFF45 by caspases during apoptosis was not observed in fractions precipitated with sWGA ( Fig. 4). These results first led to the hypothesis that the $O$-GlcNAcylation of DFF45 could protect the protein from its cleavage by caspases during apoptosis. Two serines (107 and 110) in DFF45 are located near one of the two caspase cleavage sites (117 and 224) and could represent a competitive site for O-GlcNAc modification and proteolytic cleavage [30]. However, DFF45 directly exposed to active caspase-3 was partially cleaved, suggesting that $O$-GlcNAc modifications protect DFF45 in a more complex way. The O-GlcNAcylation of DFF45 could alter its binding to other proteins or modify the chromatin structure where DFF is primarily located, rendering it less accessible to caspase-3. The $O$-GlcNAc modification has been proven to regulate chromatin dynamics through the post-translational modification of histones [31]. The largest isoform of OGA (ncOGA) possesses a HAT domain in its $\mathrm{C}$-terminal region, and the enzyme has been found in transcriptional corepression complexes along with OGT and HDACs [32]. The O-GlcNAcylation of DFF45 could also regulate its nucleocytoplasmic localization, thus explaining its protection from caspase-3 cleavage.

The role of DFF45 O-GlcNAcylation needs to be determined. It is important to assess whether the $O$-glycosylation of DFF45 can affect the binding of the protein to DFF40. It has been demonstrated that the O-GlcNAc modification of an Sp-1-derived peptide blocks its association with TAF110, providing evidence that this type of post-translational modification can influence protein-protein interactions [33]. The O-GlcNAcylation of $Y Y 1$, a transcription factor important during development, blocks its binding to the retinoblastoma protein [34]. $\mathrm{O}$ GlcNAcylation is increased in chronic lymphocytic leukemia lymphocytes [35]. The high level of $O$-GlcNAcylation correlated with the inhibition of signaling pathways controlling cell growth. Stimulation of lymphocytes with plant lectin increased nuclear $O$-GlcNAc levels and decreased cytoplasmic $O$-GlcNAc levels in T lymphocytes [36]. O-GlcNAcylation affects mature $T$ cells through the regulation of critical transcription factors. Modifications of NF-KB and NFAT by $\mathrm{O}-$ GlcNAc are required after T cell stimulation to obtain an effective response [37]. To our knowledge, this study is the first to confirm a relationship between $O$ GlcNAcylation and DNA fragmentation during apoptosis in T cells. The HPB-ALL cells were more resistant to TBT-induced nuclear apoptosis when they were treated with PUGNAc. The O-GIcNAc modification of DFF45 in normal cells may 
represent a mechanism to prevent the accidental proteolysis of this inhibitory subunit of the DFF complex.

\section{Acknowledgements}

This work was supported by a grant from NSERC: Natural Sciences and Engineering Research Council of Canada. 